\title{
STOCHASTIC STABILITY OF LINEAR SYSTEMS WITH SEMI-MARKOVIAN JUMP PARAMETERS
}

\author{
ZHENTING HOU ${ }^{1}$, JIAOWAN LUO ${ }^{2}$ and PENG SHI ${ }^{3}$
}

(Received 1 November, 2003; revised 10 March, 2004)

\begin{abstract}
Over the past two decades considerable effort has been devoted to problems of stochastic stability, stabilisation, filtering and control for linear and nonlinear systems with Markovian jump parameters, and a number of results have been achieved. However, due to the exponential distribution of the Markovian chain, there are many restrictions on existıng results for practical applications. In the present paper, we study systems whose jump parameters are semi-Markovian rather than fully Markovian. We consider only linear systems with semi-Markovian jump parameters, and also study systems with phase-type semi-Markovian jump parameters, because the family of phase-type distributions is dense in the families of all probability distributions on $[0,+\infty)$. Some stochastic stability results are obtained. An example is given to show the potential of the proposed techniques.
\end{abstract}

\section{Introduction}

In engineering applications, frequently occurring dynamical systems which can be represented by different forms depending on the value of an associated Markov chain process are termed Markovian jump systems. Research into this class of systems and their applications has spanned several decades. For some representative work on this general topic, we refer the reader to $[3,7-9,12-15,26]$ and the references therein. Some recent results can be found in $[1,2,4-6,10,16-18,21-25]$.

However, because the distribution of the Markov chain is exponential, Markovian jump systems have many limitations/restrictions in applications, and the results obtained for these systems are conservative in some senses. In this paper, our focus is

\footnotetext{
'School of Mathematics, Central South University, Changsha, Hunan 410075, China; e-mail: zthou@csu.edu.cn.

${ }^{2}$ School of Mathematics and Statistics, Carleton University, 1125 Colonel By Drive, Ottawa, Ontario KIS 5B6, Canada; e-mail: jwluo@math.carleton.ca.

${ }^{3}$ School of Technology, University of Glamorgan, Pontypridd, Wales, CF37 IDL, UK; e-mail: pshi@glam.ac.uk.
}

(C) Australian Mathematical Society 2005, Serial-fee code 1446-1811/05 
on semi-Markovian jump systems. We attempt to achieve similar results for semiMarkovian jump systems to those for Markovian jump systems. To the best of the authors' knowledge, the problems of stability, stabilisation, filtering and control for semi-Markovian jump systems have not yet been investigated.

Due to the density of phase-type distributions in the families of all probability distributions on $[0,+\infty)$, we first define the phase-type semi-Markovian process, then, for simplicity, we study the simplest linear systems with phase-type semi-Markovian jump parameters. Our results are built on the work of [8], but are more meaningful. Some stochastic stability results are obtained. The techniques employed in this paper can be applied to general control and filtering problems including nonlinear systems with semi-Markovian jump parameters.

\section{Phase-type semi-Markov processes and Markovisation}

Consider a Markov process $r(t)$ on the state space $\{1,2, \ldots, m+1\}$, where the states $1,2, \ldots, m$ are transient and the state $m+1$ is absorbing. The infinitesimal generator is

$$
Q=\left(\begin{array}{ll}
T & T^{0} \\
0 & 0
\end{array}\right)
$$

where the $m \times m$ matrix $T=\left(T_{i j}\right)$ satisfies $T_{u t}<0, T_{i j} \geq 0, i \neq j$, while $T^{0}=$ $\left(T_{1}^{0}, T_{2}^{0}, \ldots, T_{m}^{0}\right)^{T}$ is a non-negative column vector, and $T e+T^{0}=0$. The initial distribution vector is $\left(\boldsymbol{a}, a_{m+1}\right)$, where $\boldsymbol{a}=\left(a_{1}, a_{2}, \ldots, a_{m}\right), a e+a_{m+1}=1$, and $e$ denotes an $\boldsymbol{m}$-dimensional column vector having 1 's as its components.

PROPOSITION 2.1 ([19]). The distribution of the time at which $r(t)$ is absorbed in $m+1$ is

$$
F(t)=1-a \exp (T t) e, \quad t \geq 0 .
$$

We recall the following definitions.

DEFINITION 1 ([19]). The state that $r(t)$ reaches at time $t$ is called the phase of the distribution $F(\cdot)$ at time $t$. The distribution $F(\cdot)$ defined in $(2.1)$ on $[0,+\infty)$ is called a continuous phase-type $(\mathrm{PH})$ distribution and $(a, T)$ is called its representation of order $m$.

There are many $\mathrm{PH}$ distributions, for example, a negative exponential distribution is a continuous $\mathrm{PH}$ distribution, and a $k$ order Erlang distribution $E_{k}$ is also a continuous $\mathrm{PH}$ distribution. 
Similar to the study of continuous-time Markov chains, we consider the discretetime Markov chain on the state space $\{1,2, \ldots, m+1\}$, let the state $m+1$ be absorbing, and denote the transition matrix by

$$
P=\left(\begin{array}{cc}
T & T^{0} \\
0 & 1
\end{array}\right),
$$

where $T=\left(T_{i j}\right)$ is a quasi-stochastic matrix, $T_{i j} \geq 0, T e \leq e, T^{0}=(I-T) e$ is a column vector, and $I-T$ is non-singular. Define $a=\left(a_{1}, a_{2}, \ldots, a_{m}\right) \geq 0, a_{m+1} \geq 0$ and $a e+a_{m+1}=1$.

DEFINITION 2 ([19]). The discrete distribution taking values on the non-negative integer space $P_{h}$ is called a discrete $\mathrm{PH}$ distribution if and only if it is a distribution of the transition steps when the Markov chain, with transition matrix $P$ and initial distribution $\left(a, a_{m+1}\right)$, reaches the absorbing state $m+1$. Again $(a, T)$ is called its representation of order $m$.

A geometry distribution can be seen as a discrete $\mathrm{PH}$ distribution. In general, a continuous $\mathrm{PH}$ distribution and a discrete $\mathrm{PH}$ distribution are both called $\mathrm{PH}$ distributions.

PROPOSITION 2.2 ([19]). The family of PH distributions is dense in all the families of distributions on $[0,+\infty)$.

From Proposition 2.2, for every probability distribution on $[0,+\infty)$, we may choose a PH distribution to simulate the original distribution to any accuracy.

REMARK. A PH distribution is the distribution of a hitting time in a finite-state, timehomogeneous Markov chain. In 1954, Jensen [11] first introduced this distribution in an economic model, but no feasible solution was provided. The key that makes a PH distribution a powerful tool is the matrix-analysis method developed by Neuts [20] in 1975. Since the 1960 s, PH distributions have been a very effective method for analysing stochastic models in queueing theory, storage theory, reliability theory, etc. They also replace the special status of the negative exponential distribution. But many scientists and engineers are still unfamiliar with PH distributions. Very recently, it was discovered that by virtue of phase-type semi-Markov processes (defined below), the PH distribution has important applications in control theory, Markov decision theory, Markov games and stochastic differential equations. It is believed that more and more applications of the PH distribution will be discovered in many areas in the near future. This paper will show the application of the PH distribution to control theory. 
DEfinition 3. Let $E$ be a finite or countable set. A stochastic process $\hat{r}(t)$ on the state space $E$ is called a phase semi-Markov process or a denumerable phase semi-Markov process (when $E$ is finite, $\hat{r}(t)$ is also called a finite phase semi-Markov process), if the following conditions hold:

(1) The sample paths of $(\hat{r}(t), t<+\infty)$ are right-continuous step functions and have left-hand limits with probability one.

(2) Denote the $n$th jump point of the process $\hat{r}(t)$ by $\tau_{n}(n=0,1,2, \ldots)$, where $\tau_{0} \equiv 0<\tau_{1}<\tau_{2}<\cdots<\tau_{n}<\cdots, \tau_{n} \uparrow+\infty$, then $\tau_{n}(n=0,1,2, \ldots)$ are Markovian with respect to the process $\hat{r}(t)$.

(3) $F_{i j}(t)=P\left(\tau_{n+1}-\tau_{n} \leq t \mid r\left(\tau_{n}\right)=i, r\left(\tau_{n+1}\right)=j\right)=F_{i}(t)(i, j \in E, t \geq 0)$ do not depend on $j$ and $n$.

(4) $F_{1}(t)(i \in E)$ is a phase-type distribution.

Obviously, in the case when $F_{i}(t)(i \in E)$ is a negative exponential distribution, the denumerable phase semi-Markov process is a Markov chain. A denumerable phase semi-Markov process is able to overcome the restriction of the negative exponential distribution of the time that a Markov chain spends in any state. However, in much research the key problem of whether a denumerable phase semi-Markov process can replace a Markov chain or not, is equivalent to whether the denumerable phase semiMarkov process can be transformed into a Markov chain or not. And if it is a finite phase semi-Markov process, it can be transformed to a finite Markov chain. The rest of this section will show that the above claim is true.

Let $E$ be a finite or countable nonempty set, $\hat{r}(t)$ be a denumerable phase semiMarkov process on the state space $E$. Denote the $n$th jump point of the process $\hat{r}(t)$ by $\tau_{n}(n=0,1,2, \ldots)$, where $\tau_{0} \equiv 0<\tau_{1}<\tau_{2}<\cdots<\tau_{n}<\ldots$. Let $\left(a^{(i)}, T^{(l)}\right)$ $(i \in E)$ denote the $m^{(i)}$ order representation of $F_{t}(t)$, where

$$
\begin{gathered}
F_{i}(t)=P\left(\tau_{n+1}-\tau_{n} \leq t \mid \hat{r}\left(\tau_{n}\right)=i\right) \quad(i \in E), \\
a^{(i)}=\left(a_{1}^{(i)}, a_{2}^{(i)}, \ldots, a_{m^{(i)}}^{(i)}\right), \quad T^{(t)}=\left(T_{j k}^{(i)}, j, k \in E\right) .
\end{gathered}
$$

Let

$$
\begin{aligned}
p_{\imath \jmath}=P\left(\hat{r}\left(\tau_{n+1}\right)\right. & \left.=j \mid \hat{r}\left(\tau_{n}\right)=i\right) \quad(i, j \in E), \\
P & =\left(p_{i j}, i, j \in E\right), \\
(a, T) & =\left\{\left(a^{(i)}, T^{(i)}\right), i \in E\right\} .
\end{aligned}
$$

Obviously, the probability distribution of $\hat{r}(t)$ can be determined only by $\{P,(a, T)\}$.

Definition 4. $\{P,(a, T)\}$ is called the pair of a denumerable phase semi-Markov process $\hat{r}(t)$. For every $n(n=0,1, \ldots), \tau_{n} \leq t<\tau_{n+1}$, define $J(t)=$ the phase of $F_{r(t)}(\cdot)$ at time $t-\tau_{n}$. 
DEFINITION 5. The term $J(t)$ defined above is called the phase of $\hat{r}(t)$ at time $t$.

For any $i \in E$, we define

$$
\begin{aligned}
T_{j}^{(i, 0)} & =1-\sum_{k=1}^{m(i)} T_{j k}^{(i)} \quad\left(j=1,2, \ldots, m^{(i)}\right), \\
G & =\left\{\left(i, k^{(i)}\right) \mid i \in E, k^{(i)}=1,2, \ldots, m^{(i)}\right\} .
\end{aligned}
$$

From the above analysis, we can easily get the following result.

THEOREM 2.3. $Z(t)=(\hat{r}(t), J(t))$ is a Markov chain with state space $G(G$ is finite if and only if $E$ is finite). The infinitesimal generator of $Z(t)$ given by $Q=\left(q_{\mu \nu}, \mu, \nu \in G\right)$ is determined only by the pair of $\hat{r}(t)$ given by $\{P,(a, T)\}$ as follows:

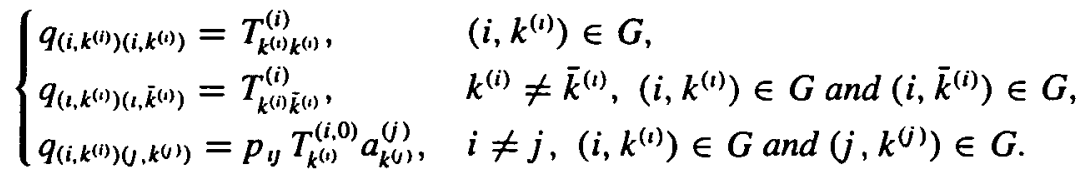

Assume that $G$ has $s=\sum_{i \in E} m^{(t)}$ elements, so the state space of $Z(t)$ has $s$ elements. We number the $s$ elements according to the following method: denote the number of $(i, k)$ by $\sum_{r=1}^{l-1} m^{(r)}+k\left(1 \leq k \leq m^{(l)}\right)$. Also denote this transformation by $\varphi$. Hence one has

$$
\varphi((i, k))=\sum_{r=1}^{i-1} m^{(r)}+k \quad\left(i \in E, 1 \leq k \leq m^{(i)}\right) .
$$

Moreover, we define

$$
\begin{aligned}
\alpha_{\varphi((i, k)) \varphi\left(\left(i^{\prime}, k^{\prime}\right)\right)} & =q_{(i, k)\left(t^{\prime}, k^{\prime}\right)} \\
r(t) & =\varphi(Z(t))
\end{aligned}
$$

Therefore $r(t)$ is a Markov chain with state space $S=\{1,2, \ldots, s\}$ and infinitesimal generator $Q=\left(\alpha_{i m}, 1 \leq i, m \leq s\right)$. We end this section by giving the following definition.

DEFINITION 6. The Markov chain $r(t)$ is called the associated Markov chain of $\hat{r}(t)$.

\section{Problem statement and main results}

Let $\{\hat{r}(t), t \geq 0\}$ be a finite phase semi-Markovian chain with state space $E$ and probability distribution $\hat{r}(t)$ which can be determined by $\{P,(a, T)\}$ as defined in 
(2.2) and (2.3). Consider a class of stochastic linear systems with semi-Markovian jump parameters in a fixed probability space $(\Omega, \mathscr{F}, P)$ :

$$
\left\{\begin{array}{l}
\dot{x}(t)=\hat{A}(\hat{r}(t)) x(t), \quad t \geq 0 \\
x(0)=x_{0}
\end{array}\right.
$$

where the initial state $x_{0}$ is a fixed nonrandom constant vector, $r(0)=r_{0}$ and $\hat{A}(i)$ $(i \in E)$ are some known matrices.

For any $i \in E, k=1,2, \ldots, m^{(i)}$, we define matrices $A$ as follows:

$$
A(\varphi(i, k)) \triangleq \hat{A}(i),
$$

where $\varphi$ is defined in (2.5).

It is easy to show that for any $(\omega, t), A(r(\omega, t)) \equiv \hat{A}(\hat{r}(\omega, t))$. Subsequently, we have the following theorem.

THEOREM 3.1. System (3.1) is equivalent to the following system:

$$
\left\{\begin{array}{l}
\dot{x}(t)=A(r(t)) x(t), \quad t \geq 0 \\
x(0)=x_{0}
\end{array}\right.
$$

where $r(t)$ is the associated Markov chain of $\hat{r}(t)$.

System (3.2) has been extensively studied in [8]. By use of Theorem 3.1 and the results of [8], we have the following main result of this paper.

THEOREM 3.2. For system (3.1),

(a) the asymptotic mean square stabilities, the exponential mean square stability and the stochastic stability are equivalent;

(b) all second moment stability imply almost sure stability;

(c) a necessary and sufficient condition for stochastic stability is that there exist positive definite matrices $M_{j}$ for $j=1,2, \ldots, s$ such that

$$
-\alpha_{i} M_{i}+\sum_{j \neq i} \alpha_{i j} M_{\jmath}+A_{i}^{\prime} M_{i}+M_{i} A_{i}=-I,
$$

where $\alpha_{i j}$ is defined in (2.6), $\alpha_{i}=-\alpha_{u}=\sum_{j \neq i} \alpha_{i j}, A_{i}=A(i)$ and $A_{1}^{\prime}$ is the transpose of $A_{i}, i, j=1,2, \ldots, s$.

REMARK. It is worth mentioning that the advantages of Theorem 3.2 are that when studying stochastic stability problems, we can replace Markovian jump systems with semi-Markovian jump systems, and get the same results, while semi-Markovian jump 
systems operate under fewer restrictions and can be widely found and used in many real system applications. Also, more importantly, almost all the nice results obtained so far for Markovian jump systems, for example, Ji and Chizeck ([12, 13]), Ji, et al. ([14]), Shi and Boukas ([21]), Shi, et al. ([22-24]), de Souza and Fragoso ([25]), and the references therein, are valid for semi-Markovian jump systems.

\section{Example}

We consider the one-dimensional case, that is, $x(t)$ takes values in $R=(-\infty,+\infty)$. Furthermore, we assume that the phase semi-Markovian process $\hat{r}(t)$ has two states denoted by 1 and 2 . The sojourn time in the first state is a random variable with negative exponential distribution with parameter $\lambda_{1}$. The sojourn in the second state is divided into two parts; the sojourn times in the two parts are two random variables which are negative exponentially distributed with parameters $\lambda_{2}$ and $\lambda_{3}$, respectively. In particular, if the process $\hat{r}(t)$ enters state 2 , it must first stay in the first part of state 2 for some time and then stay in the second part, before finally entering state 1 . We assume that $\hat{A_{1}} \neq \hat{A_{2}}$ and $p_{12}=p_{21}=1$.

Obviously,

$$
\begin{gathered}
P=\left(\begin{array}{ll}
p_{11} & p_{12} \\
p_{21} & p_{22}
\end{array}\right)=\left(\begin{array}{ll}
0 & 1 \\
1 & 0
\end{array}\right), \quad T^{(2)}=\left(\begin{array}{ll}
T_{11}^{(2)} & T_{12}^{(2)} \\
T_{21}^{(2)} & T_{22}^{(2)}
\end{array}\right)=\left(\begin{array}{cc}
-\lambda_{2} & \lambda_{2} \\
0 & -\lambda_{3}
\end{array}\right), \\
a^{(1)}=\left(a_{1}^{(1)}\right)=(1), \quad T^{(1)}=\left(T_{11}^{(1)}\right)=\left(-\lambda_{1}\right), \quad a^{(2)}=\left(a_{1}^{(2)}, a_{2}^{(2)}\right)=(1,0) .
\end{gathered}
$$

The state space of $Z(t)=(\hat{r}(t), J(t))$ is clearly $G=((1,1),(2,1),(2,2))$. We number all the elements of $G$ as follows:

$$
\varphi((1,1))=1, \quad \varphi((2,1))=2, \quad \varphi((2,2))=3 .
$$

Hence the infinitesimal generator of $\varphi(Z(t))$ is

$$
Q=\left(\alpha_{i j}\right)=\left(\begin{array}{ccc}
-\lambda_{1} & \lambda_{1} & 0 \\
0 & -\lambda_{2} & \lambda_{2} \\
\lambda_{3} & 0 & -\lambda_{3}
\end{array}\right)
$$

As $x(t) \in R$, so $A_{i}, M_{i}(i=1,2,3)$ are real constant numbers, and $A_{i}^{\prime}=A_{i}, A_{1}=\hat{A_{1}}$, $A_{2}=A_{3}=\hat{A_{2}}$. Hence (3.3) reduces to

$$
\begin{aligned}
-\lambda_{1} M_{1}+\lambda_{1} M_{2}+2 M_{1} \hat{A_{1}} & =-1, \\
-\lambda_{2} M_{2}+\lambda_{2} M_{3}+2 M_{2} \hat{A_{2}} & =-1, \\
\lambda_{3} M_{1}-\lambda_{3} M_{3}+2 M_{3} \hat{A_{2}} & =-1 .
\end{aligned}
$$


Define $\Delta=\left(\lambda_{1}-2 \hat{A}_{1}\right)\left(\lambda_{2}-2 \hat{A}_{2}\right)\left(\lambda_{3}-2 \hat{A}_{2}\right)-\lambda_{1} \lambda_{2} \lambda_{3}$. From (4.1), if $\Delta \neq 0$, then

$$
\begin{aligned}
& M_{1}=\frac{\left(\lambda_{2}-2 \hat{A}_{2}\right)\left(\lambda_{3}-2 \hat{A}_{2}\right)+\lambda_{1} \lambda_{2}+\lambda_{1}\left(\lambda_{3}-2 \hat{A}_{2}\right)}{\Delta}, \\
& M_{2}=\frac{\left(\lambda_{1}-2 \hat{A}_{1}\right)\left(\lambda_{3}-2 \hat{A}_{2}\right)+\lambda_{2} \lambda_{3}+\lambda_{2}\left(\lambda_{1}-2 \hat{A}_{1}\right)}{\Delta}, \\
& M_{3}=\frac{\left(\lambda_{1}-2 \hat{A}_{1}\right)\left(\lambda_{2}-2 \hat{A}_{2}\right)+\lambda_{1} \lambda_{3}+\lambda_{3}\left(\lambda_{2}-2 \hat{A}_{2}\right)}{\Delta} .
\end{aligned}
$$

If $\Delta=0$, then $\lambda_{1}-2 \hat{A}_{1} \neq 0, \lambda_{2}-2 \hat{A}_{2} \neq 0, \lambda_{3}-2 \hat{A}_{2} \neq 0$. Hence from (4.1) we have

$$
M_{1}=\frac{1+\lambda_{1} M_{2}}{\left(\lambda_{1}-2 \hat{A_{1}}\right)}, \quad M_{2}=\frac{1+\lambda_{2} M_{3}}{\left(\lambda_{2}-2 \hat{A_{2}}\right)}, \quad M_{3}=\frac{1+\lambda_{3} M_{1}}{\left(\lambda_{3}-2 \hat{A_{2}}\right)} .
$$

Therefore we have the following result.

(1) If $\hat{A_{1}}<0, \hat{A_{2}}<0, \hat{A_{1}} \neq \hat{A_{2}}$, then $M_{1}>0, M_{2}>0, M_{3}>0$. Then, by Theorem 3.1, system (3.1) is stable. In other words, if the original two systems are stable, then the new system, which is generated by the original two systems switched by a phase-type semi-Markovian process, is still stable.

(2) If $\hat{A}_{1}>0, \hat{A_{2}}>0, \lambda_{1}>2 \hat{A}_{1}, \lambda_{2}>2 \hat{A_{2}}, \hat{A_{1}} \neq \hat{A_{2}}$, then $M_{1}<0, M_{2}<0$, $M_{3}<0$. By Theorem 3.2, system (3.1) is not stable. In other words, if the original two systems are unstable, the new system, which is generated by the original two systems switched by a phase-type semi-Markovian process, is likely to be unstable as well.

(3) If $\hat{A}_{1}=-10, \hat{A_{2}}=3, \lambda_{1}=10, \lambda_{2}=20$, then $M_{1}=0.2851, M_{2}=0.7553$, $M_{3}=0.4787$. By Theorem 3.2, system (3.1) is stable. On the other hand, if $\hat{A}_{1}=-10, A_{2}=6, \lambda_{1}=10, \lambda_{2}=20$, then $M_{1}=-0.1654, M_{2}=-0.5962$, $M_{3}=-0.2885$. By Theorem 3.2, the new system (3.1) is unstable. Hence, if one of the two original systems is stable and the other is unstable, then the new switching system, which is generated by the original two systems switched by a phase-type semi-Markovian process, is stable or unstable depending on the parameters.

For the general situation, by Theorem 3.2 and (4.2)-(4.5), we present our last result in this paper.

COROLlaRY 4.1. Consider system (3.1) under the conditions given above.

(i) Assume that $\Delta \neq 0$, then system (3.1) is stable if and only if the following three inequalities hold:

$$
\begin{aligned}
& \Delta \times\left[\left(\lambda_{2}-2 \hat{A_{2}}\right)\left(\lambda_{3}-2 \hat{A_{2}}\right)+\lambda_{1} \lambda_{2}+\lambda_{1}\left(\lambda_{3}-2 \hat{A_{2}}\right)\right]>0, \\
& \Delta \times\left[\left(\lambda_{1}-2 \hat{A_{1}}\right)\left(\lambda_{3}-2 \hat{A_{2}}\right)+\lambda_{2} \lambda_{3}+\lambda_{2}\left(\lambda_{1}-2 \hat{A_{1}}\right)\right]>0, \\
& \Delta \times\left[\left(\lambda_{1}-2 \hat{A_{1}}\right)\left(\lambda_{2}-2 \hat{A_{2}}\right)+\lambda_{1} \lambda_{3}+\lambda_{3}\left(\lambda_{2}-2 \hat{A_{2}}\right)\right]>0 .
\end{aligned}
$$


(ii) Assume that $\Delta=0$, then system (3.1) is stable if and only if the following three inequalities hold:

$$
\left(\lambda_{1}-2 \hat{A}_{1}\right)>0, \quad\left(\lambda_{2}-2 \hat{A}_{2}\right)>0, \quad\left(\lambda_{3}-2 \hat{A}_{2}\right)>0
$$

\section{Conclusions}

In this paper, a new method has been proposed to study the problem of stochastic stability for systems with semi-Markovian jump parameters. It is a first attempt on this topic. It is shown that the existing results for stochastic stability for Markovian jump systems also hold for semi-Markovian jump systems. However semi-Markovian jump systems are less conservative and more applicable. A numerical example is given to illustrate the feasibility and effectiveness of the theoretic results obtained. The approach developed in this paper can also be applied to general nonlinear systems with semi-Markovian jump parameters.

\section{Acknowledgements}

The authors are grateful to the reviewers and Prof K. L. Teo for the valuable comments and suggestions which have improved the presentation of the paper. ZH's work was partially supported by the NNSF of China (No. 10171009), 211 Project, 985 Project, Research Fund for PhD Programs of MOE of China (No. 20010533001). J's work was supported in part by NSFC under Grant No. 10301036, and by NSERC. PS's work was partially supported by the Laboratory of Complex Systems and Intelligence Science, Institute of Automation, Chinese Academy of Sciences.

\section{References}

[1] E. K. Boukas, P. Shi and S. K. Nguang, "Robust $\mathrm{H}$-infinity control for linear Markovian jump systems with unknown nonlinearities", J. Math. Anal. Appl. 282 (2003) 241-255.

[2] O.L. V. Costa and M. D. Fragoso, "Stability results for discrete-time linear systems with Markovian jumping parameters", J. Math. Anal. Appl. 179 (1993) 154-178.

[3] M. Davis, Markov models and optimization (Chapman and Hall, London, 1992).

[4] V. Dragan, P. Shi and E. K. Boukas, "Control of singularly perturbed systems with Markovian jump parameters: an H-infinity approach", Automatica 35 (1999) 1369-1378.

[5] F. Dufour and P. Bertrand, "The filtering problem for continuous-time linear systems with Markovian switching coefficients", Syst. Control Lett. 23 (1994) 453-461.

[6] F. Dufour and P. Bertrand, "An image-based filter for discrete-time Markovian jump linear systems", Automatica 32 (1996) 241-247. 
[7] R. J. Elliott and D. D. Sworder, "Control of a hybrid conditionally linear Gaussian process", J. Optim. Theory Appl. 74 (1992) 75-85.

[8] X. Feng, K. A. Loparo, Y. Ji and H. J. Chizeck, "Stochastic stability properties of jump linear systems", IEEE Trans. Automat. Control 37 (1992) 38-53.

[9] W. Fleming, S. Sethi and M. Soner, "An optimal stochastic production planning problem with randomly fluctuating demand", SIAM J. Control Optim. 25 (1987) 1494-1502.

[10] P. Gahinet and P. Apkarian, "A linear matrix inequality approach to $H_{\infty}$ control", Int. J. Robust Nonlinear Control 4 (1994) 421-448.

[11] A. Jensen, A distribution model applicable to economics (Munkgaard, Copenhagen, 1954).

[12] Y. Ji and H. J. Chizeck, "Controllability, stabilizability and continuous-time Markovian jump linear-quadratic control", IEEE Trans. Automat. Control 35 (1990) 777-788.

[13] Y. Ji and H. J. Chizeck, "Jump linear quadratic Gaussian control: steady-state solution and testable conditions", Control Theory Adv. Tech. 6 (1990) 289-319.

[14] Y. Ji, H. J. Chizeck, X. Feng and K. A. Loparo, "Stability and control of discrete-time jump linear systems", Control Theory Adv. Tech. 7 (1991) 247-270.

[15] H. Kushner, Stochastic stability and control (Academic, New York, 1967).

[16] M. Mahmoud and P. Shi, "Robust Kalman filtering for continuous time-lag systems with Markovian jump parameters", IEEE Trans. Circuits Syst. 50 (2003) 98-105.

[17] M. S. Mahmoud and P. Shi, "Robust stability, stabilization and $H_{\infty}$ control of time-delay systems with Markovian jump parameters", J. Robust Nonlinear Control 13 (2003) 755-784.

[18] T. Morozan, "Stability and control for linear systems with jump Markov perturbations", Stochastic Anal. Appl. 13 (1995) 91-110.

[19] M. F. Neuts, "Probability distributions of phase type", in Liber Amicorum Prof., (Belgium Univ. of Louvain, 1975) 173-206.

[20] M. F. Neuts, Matrix-geometric solutions in stochastic models (John Hopkins Univ. Press, Balgium, 1981).

[21] P. Shi and E. K. Boukas, " $H_{\infty}$ control for Markovian jumping linear systems with parametric uncertainty", J. Optim. Theory Appl. 95 (1997) 75-99.

[22] P. Shi, E. K. Boukas and R. K. Agarwal, "Control of Markovian jump discrete-time systems with norm bounded uncertainty and unknown delays", IEEE Trans. Automat. Control 44 (1999) 2139-2144.

[23] P. Shi, E. K. Boukas and R. K. Agarwal, "Kalman filtering for continuous-time uncertain systems with Markovian jumping parameters", IEEE Trans. Automat. Control 44 (1999) 1592-1597.

[24] P. Shi, E. K. Boukas, S. K. Nguang and X. Guo, "Robust disturbance attenuation for discrete-time active fault tolerant control systems with uncertainties", Optimal Control Appl. Methods 24 (2003) $85-101$.

[25] C. E. de Souza and M. D. Fragoso, " $H_{\infty}$ control for linear systems with Markovian jumping parameters", Control Theory Adv. Tech. 9 (1993) 457-466.

[26] R. Srichander and B. K. Walker, "Stochastic analysis for continuous-time fault-tolerant control systems", Int. J. Control 57 (1989) 433-452. 